\title{
Cost-benefit analysis for energy storage exploitation in distribution systems
}

\author{
Gianni Celli $\bowtie$, Fabrizio Pilo, Giuditta Pisano, Gian Giuseppe Soma \\ University of Cagliari, Cagliari, Italy \\ $凶$ E-mail: celli@diee.unica.it
}

\begin{abstract}
Energy storage devices are valuable components of the future smart distribution systems, thanks to their ability to increase the flexibility of the overall system and provide a wide range of services to the distribution system operators and customers. However, their costs are still high and they still need economic incentives for their development. These incentives cannot be given to any project but only to those that demonstrate their effectiveness. This evaluation is not easy because it depends on the rate of the energy storage system device, its location on the network, and the kind of services provided. The study proposes a hybrid methodology, based on the combination of a multi-objective optimisation and a cost-benefit analysis in order to build a simple look-up table that easily allows identifying those project proposals that can be considered convenient a priori.
\end{abstract}

\section{Introduction}

Recent years have been characterised by a wide trend in the transition towards more flexible and 'smarter' distribution systems that should reduce costs, enable new services and business models, and increase the hosting capacity for renewable energy production and electric vehicles. Achieving these goals requires efficient and coordinated exploitation of new flexible resources, among which energy storage systems (ESSs) will play a key role. To optimise the expected high deployment of ESSs, a better understanding of the potential benefits is needed, and a finer decision support is expected for both system operation and planning.

The paper is based on a research project for the integration of ESSs in the distribution systems funded by the Italian Regulator (AEEGSI). Firstly, the potential services offered by the ESSs have been identified and ad hoc metrics have been formulated. Then, a planning tool for dimensioning, locating, and scheduling ESSs in realistic planning cases has been developed. To impartially assess the ESSs value, a full multi-objective (MO) approach has been adopted to consider the different point of views of the stakeholders, and to avoid assigning weights to the single objective functions and defining a priori market rules. Furthermore, an economic cost-benefit analysis (CBA) has been applied to the results of the MO optimisation in order to sort out from the Pareto front those projects that lead to an efficient use of public money.

The methodology can be used by a regulator to define the transient conditions that might allow a DSO to own and operate its own storage to fix typical network issues. The final goal is a rationale that allows the regulator to decide if a proposal for ESS can be remunerated as any other network investment. In general terms only ESS projects with a positive economic CBA can be supported, but economic CBA is a complex exercise for DSOs (particularly if small) that are much used in financial CBA. Furthermore, CBA complexity, probably too high for small projects at LV level, is not the only issue, and standardisation of calculations should not be forgotten. With the proposed methodology, possible ESS allocations are a priori analysed with a standardised procedure that populates a database of cases with high probability of success and defines clusters of representative cases. The real projects that belong to a cluster with positive CBA are assumed to be positive and could be financed without any further calculation by making the decision process as simple as looking at a look-up table.

\section{Decision-making process}

Decision making about the development of a future policy, programme or project should normally observe the following five-step process:

- identifying objectives,

- identifying options for achieving the objectives,

- defining the criteria used to compare the options,

- analysing the options, and

- making choices.

Good final choices need clear objectives that, thus, should be measurable, agreed, and realistic. The current objectives of distribution networks are the promotion of renewable energy sources (RES), the improvement or at least not worsening of service quality, and the increase of the system efficiency. To achieve these objectives, the alternatives may vary from comprehensive policies (e.g. incentive schemes for RES) to the evaluation of specific projects, such as installing ESSs in distribution networks. Once defined the criteria for comparing different alternatives, the next step of the decision-making process is the analysis of the options. Many methods exist, but basically they can be grouped into two categories: monocriteria analysis (purely monetary approach as CBA), and multi-criteria analysis (MCA) (the value of any action or result is evaluated according to specific characteristics, not necessarily economic).

\subsection{CBA approach}

The CBA is the most widely used valuation technique for comparing alternative investment projects. It is founded on two basic principles: the monetisation of the effects of a generic alternative and inter-temporal discount, used to discount future costs and benefits. The metric used for comparing costs and benefits, that must be all quantified, is always money. Once quantified and actualised future costs and benefits a given project, to be accepted, should provide total benefits exceeding the total opportunity cost of the resources used to make feasible such project (labour, time, monetary costs etc.). Despite the inherent difficulties, the quantification of intangible effects, as environmental costs, can be monetised through the willingness to pay by the potential beneficiaries of the effects, or by estimating their value using the market prices of 
tangible goods that incorporate these effects (hedonic prices method), or by using the namely shadow prices. The need to monetise each good/effect is the recognised CBA weakness. On the other hand, the $\mathrm{CBA}$ is efficient because it maximises the economic resources, objective, because it not promotes or disadvantages deliberately none of the parties, and transparent, because the monetary aspect is comprehensible also to non-experts.

\subsection{MCA approach}

The MCA is suitable for solving complex problems characterised by high uncertainty, conflicting objectives, different kinds of data, many interests and perspectives. Decisions are made based on trade-offs or compromises among a number of criteria that are in conflict with each other.

The two main groups of methods are multi-objective decision making (MODM) and multi-attribute decision making (MADM) [1]. The MODM methods are multiple objective mathematical programming models relevant for design problems, where the purpose is the identification of a preferred alternative from a potentially infinite set of alternatives implicitly defined by a set of constraints. The MADM methods are pertinent for evaluation problems whose goal is the evaluation of, and possible choice among, a limited number of predefined alternatives that are usually characterised by multiple conflicting attributes.

\subsection{Combination of MCA and CBA}

The paper focused on the development of a tool able to evaluate projects for ESSs in the distribution systems. The desired result is a decision table that defines if a given project is economically and automatically acceptable or if more project-specific investigations are necessary.

The identification of the alternatives for achieving the objectives of distribution network operation improvement has to be made. Multiple options exist depending for instance on characteristics of the network, the ESS size and location, the services offered by ESS and the ESS control logic. Thus, the design options should be derived with an optimisation capable to identify the best solutions that also have to be economically convenient. CBA or purely MCA seem is not suitable for the desired goals. CBA implies the monetisation of all benefits, and MCA the risks of selecting also those economically unacceptable as sustainable solutions (e.g. the one that leads to ESS CAPEX higher than the corresponding benefits). Therefore in this paper a hybrid decision-making approach that integrates MCA and CBA aspects has been proposed.

Firstly, an MO optimisation will be executed to identify the set of optimal (not dominated) alternatives in terms of size and position of ESS. At this stage, the evaluation criteria will be maintained with their natural metric, without performing any type of monetisation. Then, the alternatives will be analysed with CBA, by monetising the results achieved in the different used criteria. Finally, the acceptable solutions (benefits higher than costs) will be grouped into classes identified by two attributes: the size of the ESS and its position in the distribution network.

With reference to medium-voltage (MV) distribution system and the typical range of peak power and daily energy demand, thesize of ESS has been categorised into 12 classes defined in terms of nominal power and nominal duration (Table 1).

The goal in the definition of the position's classes has been the simplicity, without the need of detailed information of the power network. Thus, three characteristics have been considered: network topology, conductor type and level of distributed generation (DG) penetration. Regarding the last characteristic, it can be noted that generally DG does not cause technical constraint violations when its amount is significantly less than the local electric demand, while technical issues can become probable when the DG penetration reach and overcome the $100 \%$ of demand [2]. Thus, this threshold can be assumed as classification criteria. However, the knowledge of the demand is not immediate without ad hoc measurements. For this reason, it has been decided to refer the DG
Table 1 Classes for ESS devices in MV systems

\begin{tabular}{lcc}
\hline Size's classes & Nominal power, kW & Nominal duration, $\mathrm{h}$ \\
\hline T1 & $P_{\mathrm{ESS}} \leq 500$ & $d_{\mathrm{ESS}} \leq 5$ \\
T2 & $500<P_{\mathrm{ESS}} \leq 1000$ & $5<d_{\mathrm{ESS}} \leq 10$ \\
T3 & $d_{\mathrm{ESS}} \leq 5$ \\
T4 & $5 d_{\mathrm{ESS}} \leq 10$ \\
T5 & $1000<P_{\mathrm{ESS}} \leq 1500$ & $d_{\mathrm{ESS}} \leq 5$ \\
T6 & $5<d_{\mathrm{ESS}} \leq 10$ \\
T7 & $d_{\mathrm{ESS}} \leq 5$ \\
T8 & $1500<P_{\mathrm{ESS}} \leq 2000$ & $5<d_{\mathrm{ESS}} \leq 10$ \\
T9 & $d_{\mathrm{ESS}} \leq 5$ \\
T10 & $5<d_{\mathrm{ESS}} \leq 10$ \\
T11 & $2000<P_{\mathrm{ESS}} \leq 2500$ & $d_{\mathrm{ESS}} \leq 5$ \\
T12 & $2500<P_{\mathrm{ESS}} \leq 3000$ & $5<d_{\mathrm{ESS}} \leq 10$ \\
\hline
\end{tabular}

Table 2 Size's classes for ESS devices in MV systems

\begin{tabular}{lccc}
\hline $\begin{array}{l}\text { Position's } \\
\text { classes }\end{array}$ & $\begin{array}{c}\text { Type of } \\
\text { prevailing } \\
\text { conductor }\end{array}$ & $\begin{array}{c}\text { \% DG in the feeder } \\
\text { that includes the } \\
\text { network part with } \\
\text { ESS }\end{array}$ & $\begin{array}{c}\text { Network part with } \\
\text { ESS (its } \% \text { GD) }\end{array}$ \\
\hline A & $\begin{array}{c}\text { buried } \\
\text { overhead }\end{array}$ & $\begin{array}{c}\text { any } \\
\leq 50 \%\end{array}$ & $\begin{array}{c}\text { any } \\
\text { trunk feeder with } \\
\text { tie links and very } \\
\text { short laterals } \\
(\leq 50 \%)\end{array}$ \\
B2 & & $\begin{array}{c}\text { trunk feeder with } \\
\text { tie links and very } \\
\text { short laterals } \\
(>50 \%)\end{array}$ \\
C1 & $>50 \%$ & $\begin{array}{c}\text { laterals }(\leq 50 \%) \\
\text { laterals }(>50 \%) \\
\text { C2 }\end{array}$ \\
C3 & laterals $(\leq 50 \%)$ \\
C4 & & laterals $(>50 \%)$ \\
\hline
\end{tabular}

penetration to the sum of the nominal rate of the installed MV/LV transformers. By assuming an average transformer loading of 50\%, the threshold considered for identifying the position's classes of ESS has been $50 \%$ of the sum of all the MV/LV transformer rates in the portion of the distribution network examined. The position's classes identified are summarised in Table 2.

The combination of these two attributes defines the categories for the classification of ESS alternatives produced by the MO optimisation.

\section{MO optimisation}

In the paper, the non-dominated sorting genetic algorithm (NSGA-II) has been implemented to find the optimal set of ESS allocations and sizes in a given distribution network. The keystone of its recognised outperformance is the two-step classification procedure used to compare individuals in each population. The first step groups the solutions into different fronts of non-dominance: each individual is non-dominated by any other in the same front but dominates every individual in the fronts of lower rank. The second step is used to preserve diversity in each Pareto front. A crowding distance attribute is defined to estimate the density of solutions around each individual in the front: solutions with high crowding distance are those located in the less crowded regions of the front and hence they are considered better than the others. The higher value of this attribute is given to the boundary solutions of the front (the best ones with respect to each objective function).

These two attributes are used to rank the individuals in a population, allowing its evolution through the application of the three genetic operators of selection, crossover and mutation. Furthermore, they are also used to rank the set of generated offspring and guarantee elitism through the comparison with the parents and the selection of only the better individuals to form the new population. 


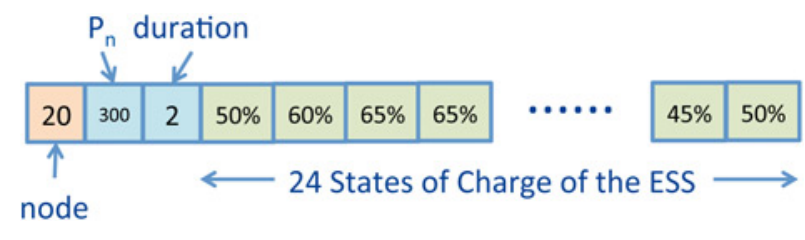

Fig. 1 Chromosome representation of an individual

\subsection{Adaptation of the MO optimisation for the ESS allocation problem}

The traditional binary coding of the genetic algorithms makes them particularly suitable for solving facility allocation problems. In the research field of power distribution system, they have been used extensively for siting and sizing many types of equipment like generators, capacitor banks, measurement and control devices, and in the last decade also ESSs.

For energy storage devices the problem is even more complex, because the diverse benefits they provide can depend not only on its rate and location but also on its daily scheduling (e.g. energy losses reduction, voltage regulation, peak shaving). Typically, in literature this issue has been faced by using a single-objective formulation (cost minimisation) and a single ESS's benefit (e.g. arbitrage) [3] or by splitting it into two nested optimisation problems: the siting and sizing of ESSs as main optimisation, and the scheduling as secondary optimisation [4].

In the paper, in order to preserve the MO approach and to find not only the best daily energy scheduling of the ESSs for each objective function but also some compromise scheduling, the chromosome of the generic solution has been organised for including not only the position and the rate (nominal power and duration) of the ESS but also its daily energy scheduling, as depicted in Fig. 1.

Four types of information have been coded in the chromosome of each allocated ESS:

(i) one position gene, whose allele indicates the distribution network node of the ESS connection,

(ii) one gene for the nominal power of the ESS, expressed in $\mathrm{kW}$, (iii) one gene for the nominal duration of the ESS, expressed in hours,

(iv) 24 genes for the levels of energy stored in the device (state of charge - SoC) at the beginning of the $24 \mathrm{~h}$ in the typical day of the year, expressed as percentage of the nominal capacity of the battery.

Considering that, apart from the position gene, all the other information can be represented by continuous variables, a real coding has been adopted in the NSGA-II, in substitution of the traditional discretisation of the states that each gene can assume.

During the evolution process, each allele has to remain in its feasible range. Moreover, two additional constraints have to be satisfied by the SoC genes:

- the difference between the alleles of two successive genes (consecutive hours) cannot exceed the nominal charging/ discharging power of the battery,

- the previous constraint has to be verified also between the first and the last genes, in order to make the scheduling repeatable for all the days in a year.

\section{Evaluation criteria}

Several evaluation criteria can be considered for the analysis of ESS allocation projects. In the paper, a simplified study has been conducted by selecting only the network investment, the energy losses, the reactive power exchange with TSO and the ESS cost.

\subsection{Network investment}

The network investments are assessed as

$$
C_{U}=\sum_{j=1}^{N_{\text {branches }}} C_{0 j}=\sum_{j=1}^{N_{\text {branches }}}\left(B_{0 j}+M_{0 j}-R_{0 j}\right)
$$

where $N_{\text {branches }}$ is the number of network branches, $C_{0 j}$ is the present cost of the $j$ th branch, and $B_{0 j}, M_{0 j}$, and $R_{0 j}$, are respectively its building, management and residual costs transferred to the cash value at the beginning of the planning.

\subsection{Energy losses}

The customer's demand variation has been modelled as a piecewise linear curve, with the load growth rate that may be different in each sub-periods into which the whole planning period has been divided. Owing to this statement, it is acceptable for planning studies to assume that the branch current grows linearly, making easy the assessment of the Joule energy losses for the $j$ th branch in the $k$ th sub-period through the following expression (in $\mathrm{kWh}$ ):

$$
E_{\mathrm{L} j k}=\frac{3.8760}{1000} R_{j} \int_{0}^{N_{K}} I_{j k}^{2} \mathrm{~d} y=26.28 R_{j} N_{k}\left(I_{f i k}^{2}+I_{0 j k}^{2}+I_{f i k} I_{0 j k}\right)
$$

where $I_{0 j k}$ and $I_{f j k}$ are respectively the branch current at the beginning and at the end of the sub-period, $N_{k}$ is the sub-period duration in years, and $R_{i}$ is the branch resistance. The total energy losses, $E_{\mathrm{L}}$, are then obtained by summing the contributions of all branches in each sub-period.

\subsection{Reactive power exchange with the TSO}

The presence of the interface inverter in the ESS device can be used to separate the exchange of active and reactive power. This aspect can be used to manage the flows of reactive power in the high-voltage side of the primary substations and limit the costs that the distributors have to pay. Indeed, in the case of absorption of reactive power $>50 \%$ of the active power, the DSO has to pay a penalty, according to Italian regulation framework (resolution 654/ $2015 / \mathrm{R} / \mathrm{EEL}$ ). In particular, during peak hours, if the reactive power is between 50 and $75 \%$ of the active power, the cost is $1.510 \mathrm{c} € / \mathrm{kvarh}$, while if it exceeds the $75 \%$, the cost becomes $1.890 \mathrm{c} € /$ kvarh.

\subsection{Cost of the energy storage system}

The cost of the ESSs has been evaluated with a similar approach adopted for the network investment, considering a life time of 10 years and the following specific costs: $200-400 € / \mathrm{kWh}$.

\section{Results and discussion}

The described procedure for the analysis of possible ESS allocation projects in MV distribution systems has been applied to the Italian representative networks defined in the project ATLANTIDE [5]. In particular, two networks have been used: urban and rural representative networks. Both networks have been studied with two scenarios of DG penetration: a business-as-usual scenario and a development scenario, with high diffusion of generation. The network calculations have been executed with a probabilistic load flow in each time step used to discretise the daily profiles of load and generators and for every network configuration in ordinary and emergency conditions ( $N-1$ analysis). The results are the probability distributions of branch currents and nodal voltages used to check the risk to violate the technical constraints [6]. The period taken into account for the planning studies is 10 years. For each MV/LV node a constant power demand growth rate of $3 \%$ 


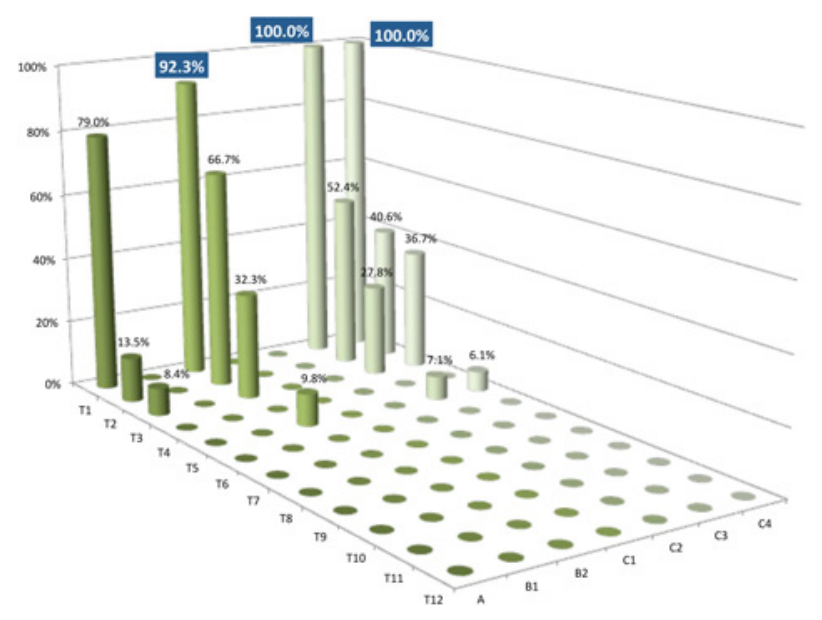

Fig. 2 Distribution of alternatives with positive CBA

per year has been assumed. Each load and each generator has been modelled with typical daily profiles that take account of the uncertainties in the demand or production by means of a normal probabilistic distribution function.

The MO optimisation has been executed in the four networks, limiting to two devices the maximum number of allocable ESS. Altogether, more than 10.000 non-dominated alternatives have been produced, differentiated by number, location, rate and daily schedule.

The subsequent societal CBA analysis applied to these solutions has pointed out that the best ones are those characterised by a single ESS device. In general terms, the configurations that produce more benefits than costs are those with a good compromise among the different objective functions (particularly in terms of daily schedule) and with a limited ESS energy rate.

Around $6 \%$ of the Pareto solutions resulted acceptable after the CBA analysis. By classifying these alternatives into the categories defined in Tables 1 and 2, the histogram of Fig. 2 is realised. The preferable locations for the ESS are the trunk feeders with high penetration of DG (class B2) and the laterals with any penetration of DG, supplied by feeders with high penetration of DG (classes $\mathrm{C} 3$ and $\mathrm{C} 4$ ). The preferred rates are all characterised by ESS with low-energy content (classes $\mathrm{T} 1, \mathrm{~T} 2$ and $\mathrm{T} 3$ ). By assuming that a category of ESS projects is acceptable if at least $80 \%$ of the Pareto solutions within this category have a positive CBA, the final look-up table is represented in Table 3. In conclusion, the results obtained have pointed out that the convenient solutions are characterised by small ESS rates (nominal power lower than $500 \mathrm{~kW}$ and nominal duration lower than $5 \mathrm{~h}$ ), with the device allocated in rural networks and high penetration of DG. Thus, a DSO that would submit a project for ESS installation could check if the portion of the network involved in the project falls into one of the a priori acceptable categories identified in Table 3 (yes cells). If the project does not fall in a positive cell, the DSO could prove the worth of the proposal by doing its own studies. To check the validity of the final look-up table, the same procedure has also been applied to some real networks (different from the representative ones). The hypothesis is that the DSO can use the proposed methodology to assess the convenience of an ESS allocation project on its MV distribution system, creating its own look-up table. The results have shown that the comparison between the DSO look-up table and the one derived with the representative networks is positive in the $88 \%$ of the time. The majority of the differences are concentrated in the urban areas classified as class A. Therefore, a better clustering procedure with
Table 3 Look-up table for ESS allocation on MV systems

\begin{tabular}{llllllll}
\hline & A & B1 & B2 & C1 & C2 & C3 & C4 \\
\hline T1 & no & no & yes & no & no & yes & yes \\
T2 & no & no & no & no & no & no & no \\
T3 & no & no & no & no & no & no & no \\
T4 & no & no & no & no & no & no & no \\
T5 & no & no & no & no & no & no & no \\
T6 & no & no & no & no & no & no & no \\
T7 & no & no & no & no & no & no & no \\
T8 & no & no & no & no & no & no & no \\
T9 & no & no & no & no & no & no & no \\
T10 & no & no & no & no & no & no & no \\
T11 & no & no & no & no & no & no & no \\
T12 & no & no & no & no & no & no & no \\
\hline
\end{tabular}

the identification of more than one class is necessary in order to improve the quality of results.

\section{Conclusions}

The regulation for enabling DSO to own and operate storage is still on the way. New EU directives (winter package 2016) do allow DSO own storage and derogations are allowed under strict conditions. EES can be remunerated only if CBA is positive (for the society).

The Italian regulator (AEEGSI) financed a research project for finding the conditions that can entitle DSO to own storage as regulated bodies and obtain the remuneration of investments. A complex methodology based on MO genetic algorithms, CBA, and clustering techniques has been developed and presented in this paper. The more complex the methodology the simpler is the application. Indeed, it will be as simple as using a look-up table. First results showed that by looking only to distribution services, only few cases exist where DES is more convenient than other investments and only in small scale (nominal power lower than $500 \mathrm{~kW}$ and nominal duration lower than $5 \mathrm{~h}$ ), with the device allocated in rural networks with high penetration of DG.

Future research are required for the improvement of distribution network clustering, particularly in the urban context, where the sensitivity analysis of most significant parameters and the inclusions of benefits are not considered.

\section{Acknowledgments}

The authors warmly thank AEEGSI for funding this research activity with a research project that might help define the future regulatory framework for ESS owned by DSO. Particularly, the authors would like to mention the valuable contribution of Dr. L. Lo Schiavo and Dr. S. Larzeni from AEEGSI for setting up the procedure and tuning the relevant metrics.

\section{References}

1 Zhou, P., Ang, B. W., Poh, K.L.: 'Decision analysis in energy and environmental modelling: an update', Energy, 2006, 31, pp. 2604-2622

2 Celli, G., Pilo, F., Soma, G.G., et al.: 'Active distribution network cost/benefit analysis with multi-objective programming'. Proc. CIRED Conf., Prague, 2009

3 Hatziargyriou, N. D., Skrlec, D., Capuder, T., et al.: 'Review of energy storage allocation in power distribution networks: application, methods and future research', IET Gener. Transm. Distrib., 2015, 10, pp. 645-652

4 Carpinelli, G., Celli, G., Mocci, S., et al.: 'Optimal integration of distributed energy storage devices in smart grids', IEEE Tram Smart Grid, 2013, 4, pp. 985-995

5 ATLANTIDE project. www.progettoatlantide.it

6 Celli, G., Ghiani, E., Pilo, F., et al.: 'New electricity distribution network planning approaches for integrating renewable', IVIREs Energy Environ., 2013, 2, pp. $140-157$ 